\section{Confiabilidade do informante secundário em inquéritos de saúde}

\author{
Renata Jardim* \\ Sandhi Maria Barreto** \\ Luana Giatti Gonçalves***
}

Informações em saúde são fundamentais para conhecer perfis epidemiológicos e demográficos, analisar estatísticas vitais e estudos socioeconômicos, para o embasamento de pesquisas e planejamento de políticas públicas. No Brasil, os Sistemas Nacionais de Informação em Saúde gerenciados pelo Sistema Único de Saúde, tais como o Sistema de Informações sobre Mortalidade (SIM), de doença de notificação compulsória e outros agravos (Sinan) e de nascidos vivos (Sinasc) são responsáveis pela maioria dos dados existentes em saúde para a população brasileira (MALTA; LEAL; COSTA; NETO, 2008).

Outra fonte de dados em saúde advém dos inquéritos nacionais realizados pelo IBGE (Instituto Brasileiro de Geografia e Estatística), nos quais a PNAD (Pesquisa Nacional por Amostra de Domicílios) e seus suplementos de saúde se destacam como o inquérito domiciliar de base populacional de maior abrangência e representatividade nacional (MALTA; LEAL; COSTA; NETO, 2008).

A PNAD é realizada no Brasil desde 1967, com periodicidade anual a partir de 1971 e abrangência nacional desde 2004. Investigam-se informações sobre características demográficas, habitação, educação, trabalho e rendimentos da população brasileira. Outros temas são incluídos com periodicidade variável, conforme necessidades vigentes. Em 1981, 1986, 1998, 2003 e 2008 foi incorporado um suplemento especial sobre saúde. Ou seja, a partir de 1998, a saúde passou a ser investigada quinquenalmente (TRAVASSOS; VIACAVA; LAGUARDIA, 2008).

O tamanho da amostra e a quantidade de informações coletadas permitem análises separadas de subgrupos (faixa etária, sexo, raça/cor, entre outros) e de associações seccionais entre diversos eventos em saúde e importantes determinantes sociais do processo saúde-doença, bem como a investigação de tendências temporais de vários indicadores, tais como uso de serviços de saúde e algumas morbidades referidas.

A PNAD tem como unidade amostral o domicílio e prevê o uso de respondentes substitutos (VIACAVA, 2002). Em 1998, 74\% dos homens e $54 \%$ das mulheres tiveram seu próprio estado de saúde avaliado por informantes secundários e, no caso das muIheres, a avaliação foi pior quando feita por outra pessoa do domicílio (VIACAVA, 2006). Entretanto, não se sabe se as informações obtidas por este meio introduzem erros de mensuração nos resultados observados.

Outros inquéritos de saúde também incluem informações coletadas de informantes substitutos, a fim de se obter um tamanho amostral suficiente para realizar diferentes análises com adequado poder estatístico (NELSON; LONGSTRETH Jr.; KOEPSELL; VAN BELLE, 1990; SHIELDS, 2000). Apesar das vantagens do uso de informantes secundários, alguns estudos têm mostrado que a concordância entre as informações obtidas diretamente do indivíduo e de um proxy varia e é influenciada por fatores como a natureza da pergunta, a idade e a escolaridade do informante secundário (FRAGA-MAIA; SANTANA, 2005; MILNE et al., 2006; PICKARD; KNIGHT, 2005; MAGAZINER et al., 1996).

\footnotetext{
* Fonoaudióloga, doutoranda em Saúde Pública, Programa de Pós-Graduação em Saúde Pública da Universidade Federal de Minas Gerais. Faculdade de Medicina.

** Médica, doutora em Epidemiologia, Programa de Pós-Graduação em Saúde Pública da Universidade Federal de Minas Gerais. Faculdade de Medicina.

${ }^{* \star *}$ Médica, doutora em Saúde Pública, Programa de Pós-Graduação em Saúde Pública da Universidade Federal de Minas Gerais. Faculdade de Medicina.
} 
$\mathrm{Na}$ tentativa de controlar o efeito de possíveis vieses introduzidos pelo uso de informante secundário, alguns artigos têm incluído a variável "respondido por proxy" na análise multivariada (DACHS, 2002; BARROS; CESAR; CARANDINA; TORRE, 2006), ou têm analisado separadamente as respostas dadas pelo sujeito índice e pelo informante secundário, comparando o resultado destas análises para avaliar possíveis diferenças nas associações investigadas (LIMA-COSTA et al., 2007; TAVARES; GUIDERRI, 2008). Porém, essas estratégias não permitem afirmar se a informação obtida pelo informante substituto introduz viés e, ainda, qual a magnitude e direção desse possível viés.

Existem inúmeros trabalhos importantes em saúde que utilizam os bancos de dados da PNAD (BARROS et al., 2006; ALMEIDA et. al., 2002; TORRES; BICHIR; CARPIM, 2006; LIMA-COSTA; BARRETO; GIATTI, 2003), como o estudo sobre as desigualdades sociais e morbidade referida que utilizou dados da PNAD 2003 (BARROS et al., 2006). Neste estudo, a maioria dos homens de 20 a 59 anos teve sua entrevista respondida por outra pessoa. A proporção de respostas obtidas de um proxy diferiu entre os sexos e entre as diversas faixas etárias. Não se sabe como a utilização de outro respondente influenciou a mensuração das informações sobre saúde na população estudada.

A confiabilidade das informações fornecidas pelo proxy necessita ser mais bem investigada. Uma estimativa de confiabilidade comumentemente utilizada em escalas nominais é o kappa de Cohen, que fornece uma medida quantitativa da magnitude de concor- dância entre os observadores, além daquela verificada pela chance. A variação interobservador pode ser mensurada em qualquer situação na qual dois ou mais observadores independentes avaliam o mesmo objeto ou assunto. O cálculo do kappa baseia-se na diferença entre a concordância observada e a concordância esperada apenas pela chance (VIERA; GARRET, 2005) (Quadro 1).

Um aspecto relevante do kappa é que sua medida pode sofrer a influência da prevalência do fenômeno estudado, particularmente para eventos raros (VIERA; GARRET, 2005). Nestes casos, baixos valores de kappa não refletem necessariamente baixas taxas de concordância total. Para lidar com este paradoxo, pode-se utilizar o kappa ajustado pela prevalência (MAK; YAU; CHAN, 2004; RAO; MILLER; RAO, 2007), que minimiza o efeito da prevalência na estimativa, pois avalia a proporção de concordância entre as variáveis menos a proporção de desacordos, valorizando a concordância observada. O cálculo deste kappa pode ser expresso por 2Po-1 ou Po-(1-Po), onde Po é a proporção da concordância observada (RAO; MILLER; RAO, 2007). Outra opção ao utilizar o kappa é aliá-lo à análise da homogeneidade das probabilidades marginais, o que permite inferir se as estimativas do kappa são adequadas. Quando as probabilidades marginais são similares, o kappa produz resultados semelhantes a outros estimadores (SLATER, 2006).

Outra medida que auxilia a investigação da reprodutibilidade de informações é o Viés Proporcional, que é dado por VP $=\left(\mathrm{P}_{\mathrm{px}}-\mathrm{P}_{\mathrm{pr}}\right) /$ $P_{p x}$, onde $P_{p x}$ é a proporção de respostas

QUADRO 1

Cálculo do Kappa de Cohen

\begin{tabular}{|c|c|c|c|c|}
\hline & & \multicolumn{3}{|c|}{ Observador 1} \\
\hline \multirow{4}{*}{ 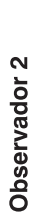 } & & Classificação A & Classificação B & Total \\
\hline & Classificação A & a & $b$ & $m_{1}$ \\
\hline & Classificação B & c & $d$ & $m_{0}$ \\
\hline & Total & $\mathrm{n}_{1}$ & $\mathrm{n}_{0}$ & $\mathrm{n}$ \\
\hline
\end{tabular}

Concordância observada $\left(p_{0}\right)=a+d / n$

Concordância esperada $\left(\mathrm{p}_{\mathrm{e}}\right)=[(\mathrm{n} 1 / \mathrm{n})$ * $(\mathrm{m} 1 / \mathrm{n})]+[(\mathrm{no} / \mathrm{n})$ * $(\mathrm{mo} / \mathrm{n})]$

$\operatorname{Kappa}(\mathrm{K})=\left(\mathrm{p}_{\mathrm{o}}-\mathrm{p}_{\mathrm{e}}\right) /\left(1-\mathrm{p}_{\mathrm{e}}\right)$

Fonte: Modificado de Viera e Garret (2005). 
positivas dadas pelos informantes primários e $\mathrm{P}_{\mathrm{pr}}$ a proporção de respostas positivas de acordo com os informantes secundários. Valores negativos representariam uma subestimação, enquanto valores positivos corresponderiam a uma superestimação da medida obtida de informantes secundários em comparação àquela obtida do informante primário (SANTANA et al., 1997).

Outra possibilidade de se investigar a confiabilidade de informantes substitutos pode ser por meio da comparação de resultados de regressão logística em informantes primários e seus respectivos informantes secundários.

Considerando o uso do informante secundário em inquéritos como a PNAD e o desconhecimento da confiabilidade de suas informações, evidencia-se a urgência de se analisarem a concordância e os demais fatores relacionados no relato de exposições e eventos referentes à saúde. Para auxiliar a preencher essa lacuna, um inquérito de saúde de base populacional, coordenado pelas autoras, realizou um conjunto de perguntas iguais ou similares à da PNAD em 710 pares de informantes primários-secundários. A comparação das respostas obtidas permite ampliar o conhecimento sobre a influência do informante secundário nos dados coletados.

\section{Referências}

ALMEIDA, M.F.; BARATA, R.B.; MONTERO, C.V.; SILVA, Z.P. Prevalência de doenças crônicas auto-referidas e utilização de serviços de saúde, PNAD/1998, Brasil. Ciência \& Saúde Coletiva, 7(4):743-756, 2002.

BARROS, M.B.A.; CESAR, C.L.G.; CARANDINA, L.; TORRE, G.D. Desigualdades sociais na prevalência de doenças crônicas no Brasil, PNAD-2003. Ciência \& Saúde Coletiva, 11(4):911-926, 2006.

DACHS, J.N.W. Determinantes das desigualdades na auto-avaliação do estado de saúde no Brasil: análise dos dados da PNAD/1998. Ciência \& Saúde Coletiva, 7(4):641-657, 2002.

FRAGA-MAIA, H.; SANTANA, V.S. Concordância de informações de adolescentes e suas mães em inquérito de saúde. Rev. Saúde Pública, 39(3):430-7, 2005.
A análise dos dados preliminares indica a ocorrência de vieses de mensuração, especialmente em variáveis mais subjetivas, como a avaliação geral de saúde. O estudo também incluiu duas questões relevantes para a compreensão da discordância observada entre indivíduos primários e secundários: uma sobre o grau de conhecimento que o informante secundário julga ter sobre a saúde do informante primário; e outra que compara a resposta do proxy quando este responde segundo sua própria perspectiva e quando o mesmo adota o ponto de vista do informante primário. A explicitação da perspectiva da resposta obtida e a pergunta que permite inferir o grau de conhecimento do informante secundário sobre a saúde do informante primário podem ser mais úteis em análises posteriores do que o "ajuste pelo proxy" nas análises multivariadas.

Essa nota objetiva ampliar a discussão e fomentar investigações sobre o uso de informantes substitutos em estudos de saúde e sua repercussão na qualidade dos dados coletados, além de referenciar alguns indicadores de confiabilidade que podem ser utilizados nos estudos que estimem a confiabilidade do informante secundário.

LIMA-COSTA, M.F.; BARRETO, S.M.; GIATTI, L. Condições de saúde, capacidade funcional, uso de serviços de saúde e gastos com medicamentos da população idosa brasileira: um estudo descritivo baseado na Pesquisa Nacional por Amostra de Domicílios. Cad. Saúde Pública, 19(3):735-743, 2003.

LIMA-COSTA, M.F.; PEIXOTO, S.V.; MATOS, D.L.; FIRMO, J.O.A.; UCHÔA, E. A influência de respondente substituto na percepção da saúde de idosos: um estudo baseado na Pesquisa Nacional por Amostra de Domicílios $(1998,2003)$ e na coorte de Bambuí, Minas Gerais, Brasil. Cad. Saúde Pública, Rio de Janeiro, 23(8):1.893-1.902, ago. 2007.

MAGAZINER, J.; BASSET, S.S.; HEBEL, J.R.; GRUBER-BALDINI, A.L. Use of proxies to measure health and functional status in epidemiologic studies of community-dwelling women aged 65 years and older. American 
Journal of Epidemiology, 143(3):283-292, 1996.

MAK, H.K.F.; YAU, K.K.W.; CHAN, B.P.L. Prevalence-adjusted Bias-adjusted. Values as Additional Indicators to Measure Observer Agreement [letter]. Radiology, v. 232 (1): 302-3, 2004.

MALTA, D. C.; LEAL, M. C.; COSTA, M. F. L.; NETO, O.L.M. Inquéritos nacionais de saúde: experiência acumulada e proposta para o inquérito de saúde brasileiro. Rev. Bras. Epidemiol.,11(supl. 1): 159-67, 2008.

MILNE, D.J.; MULDER, L.L.; BEELEN, H.C.M.; SCHOEFIELD, P.; KEMPEN, G.I.J.M.; ARANDA, S. Patients' self-reporting and family caregivers' perception of quality of life in patients with advanced cancer: how do they compare? European Journal of Cancer Care, 15:125-32, 2006.

NELSON, L.M.; LONGSTRETH Jr., W. T.; KOEPSELL, T.T.; VAN BELLE, G. Proxy respondents in epidemiologic research. Epidemiologic Reviews, 12:71-86, 1990.

PICKARD, A.S.; KNIGHT, S.J. Proxy evaluation of health-related quality or life - a conceptual framework for understanding multiple proxy perspectives. Medical Care, 43(5): 493-9, 2005.

RAO, C.R.; MILLER, J.P.; RAO, D.C. Handbook of statistics 27 - Epidemiology and Medical Statistics, 2007, p.115-6.

SANTANA, V.S.; ALMEIDA FILHO, N.; ROCHA, C.O.; MATOS, A.S. Confiabilidade e viés do informante secundário na pesquisa epidemiológica: análise de questionário para triagem de transtornos mentais. Rev. Saúde Pública, 31(6): 556-65, 1997.
SHIELDS, M. Proxy reporting in the National Population Health Survey. Health Reports, 12(1): 21-39, 2000. Disponível em: <http:// www.statcan.gc.ca/studies-etudes/82-003/ archive/2000/5300-eng.pdf>. Acesso em: 24 out. 2006.

SLATER, M.B. Concurrent assessment of interrater agreement and intrarater reliability in the case of binay data. Dissertação de Mestrado. Calgary: Universidade de Calgary, 2006.

TAVARES, D.M.S.; GUIDERRI, G.C.B.; SAÚDE, M.I.B.M. Características sócio-demográficas, condições de saúde e utilização de serviços de saúde por idosos. Revista Eletrônica de Enfermagem, 10(2):299-309, 2008. [Internet].

TORRES, H.G.; BICHIR, R.M.; CARPIM, T.P. Uma pobreza diferente? Mudanças no padrão de consumo da população de baixa renda. Novos Estudos, Cebrap, 74:17-22, 2006.

TRAVASSOS, C.; VIACAVA, F.; LAGUARDIA, $J$. Os suplementos saúde na Pesquisa Nacional por Amostra de Domicílios (PNAD) no Brasil. Rev. Bras. Epidemiol., 11(supl. 1): 98-112, 2008.

VIACAVA, F. Informações em saúde: a importância dos inquéritos populacionais. Ciênc. Saúde Coletiva, v.7(4):607-21, 2002.

VIACAVA, F.; DACHS, N.; TRAVASSOS, C. Os inquéritos domiciliares e o Sistema Nacional de Informações em Saúde. Ciência \& Saúde Coletiva, 11(4):863-869, 2006.

VIERA, A.J.; GARRET, J.M. Understanding Interobserver Agreement: the Kappa Statistic. Family Medicine, v. 37 (5):360-3, 2005.

Recebido para publicação em 16/04/2009. Aceito para publicação em 25/04/2009. 